Research Article

\title{
Severity, Treatment, and Outcome of Acute Pancreatitis in Thailand: The First Comprehensive Review Using Revised Atlanta Classification
}

\author{
Supot Pongprasobchai, ${ }^{1}$ Peeradon Vibhatavata, ${ }^{2}$ and Piyaporn Apisarnthanarak ${ }^{3}$ \\ ${ }^{1}$ Division of Gastroenterology, Department of Medicine, Faculty of Medicine Siriraj Hospital, Mahidol University, \\ Bangkok, Thailand \\ ${ }^{2}$ Department of Medicine, Faculty of Medicine Siriraj Hospital, Mahidol University, Bangkok, Thailand \\ ${ }^{3}$ Division of Diagnostic Radiology, Department of Radiology, Faculty of Medicine Siriraj Hospital, Mahidol University, \\ Bangkok, Thailand
}

Correspondence should be addressed to Supot Pongprasobchai; supotpong@gmail.com

Received 25 September 2016; Revised 24 February 2017; Accepted 20 March 2017; Published 13 April 2017

Academic Editor: Atsushi Irisawa

Copyright (c) 2017 Supot Pongprasobchai et al. This is an open access article distributed under the Creative Commons Attribution License, which permits unrestricted use, distribution, and reproduction in any medium, provided the original work is properly cited.

\begin{abstract}
Background. Severity and outcome of acute pancreatitis (AP) in Thailand are unknown. Methods. A retrospective study of 250 patients with AP during 2011-2014 was performed. Severity, treatment, and outcome were evaluated. Severity was classified by revised Atlanta classification. Results. The mean age was 58 years and 56\% were men. Etiologies were gallstones (45\%), alcohol (16\%), postendoscopic retrograde cholangiopancreatography (14\%), and idiopathic (15\%). Overall, $72 \%, 16 \%$, and $12 \%$ of patients had mild, moderately severe, and severe AP, respectively. Two major types of initial intravenous fluid were normal saline (64\%) and Ringer's lactate solution (RLS, 28\%). Enteral nutrition was given in $77 \%$ of patients with severe AP, median duration 48 hours, and via a nasogastric tube in $67 \%$ of patients. Necrotizing pancreatitis (NP) developed in $7 \%$ of patients, and $29 \%$ of them developed infection (median 17 days). The median length of stay was 6, 9, and 13 days, and the mortality rate was $1 \%, 3 \%$, and $42 \%$ in mild, moderately severe, and severe AP, respectively. The overall mortality rate was $6 \%$. Conclusion. The severity of AP in Thailand was mild, moderately severe, and severe in $72 \%, 16 \%$, and $12 \%$ of patients, respectively. NP was not prevalent. Mortality was high in severe AP. Most treatments complied with standard guidelines except the underuse of RLS.
\end{abstract}

\section{Introduction}

Acute pancreatitis (AP) is an acute pancreatic inflammatory process triggered by abnormal activation of pancreatic enzymes and the release of several inflammatory mediators. The disease exhibits variable involvement of other regional tissues and remote organ systems [1]. The overall mortality rate of $\mathrm{AP}$ is approximately 5\% [2]. There is a wide variation in the disease severity, ranging from mild, self-limiting disease that recovers within 3 to 5 days without mortality to severe, life-threatening disease with a mortality rate of $30 \%$ to $40 \%$ [3].
The Atlanta classification of 1992 provided a global consensus and universally applicable classification system for AP [1]. Although it is useful and widely accepted, some of the definitions used in this version of the classification remain confusing. A better understanding of the pathophysiology of organ failure (OF) and necrotizing pancreatitis (NP) and their outcomes, as well as improvements in diagnostic imaging techniques, has made it necessary to revise the Atlanta classification. The 2012 revision of the Atlanta classification includes clinical assessment of the severity of AP as mild, moderately severe, or severe and provides more objective terms with 
which to describe the local complications of AP. Thus, it is currently the gold standard for evaluating the severity of AP [4].

In Thailand, studies on AP are very scarce despite the importance of the disease [5-8]; this lack of data is partly due to the uncommonness of AP. Basic information on the severity and outcomes of AP in Thai patients using the current classification would represent a milestone in the development of a Thai guideline for AP; however, such data are lacking. In the present study, we evaluated the severity, treatment, and outcomes of patients with AP in our institution according to the 2012 revision of the Atlanta classification.

\section{Materials and Methods}

2.1. Study Design and Population. This retrospective study involved all adult patients who were admitted to Siriraj Hospital, Bangkok, Thailand, for the treatment of AP from December 2011 to December 2014. The study was approved by the Siriraj Institutional Review Board.

All adult patients aged $\geq 18$ years who were diagnosed with AP by meeting two of the three diagnostic criteria for AP [abdominal pain, a serum amylase or lipase activity three times higher than the upper limit of normal, and pancreatitis documented by computed tomography (CT)] were enrolled $[1,4]$. Only patients who initially presented to our hospital within 48 hours were included in the study. Patients with incomplete medical records, especially in the first 48 hours of disease, were excluded.

2.2. Clinical Data. Data regarding severity, treatment, and outcomes of AP were collected using designed case record forms.

2.3. CT Findings. All CT scans were reviewed by two of the authors: a gastroenterologist with expertise in pancreatic diseases (S.P.) and a specialized gastrointestinal radiologist (P.A.). The terminology used followed the 2012 revision of the Atlanta classification [4].

2.4. Definitions of Severity of AP and Local Complications. The severity of AP and terminology regarding systemic and local complications were classified according to the 2012 revision of the Atlanta classification [4].

2.5. Statistical Analysis. Descriptive statistics were used to summarize the patients' characteristics. Continuous data are presented as mean and standard deviation, whereas categorical data are presented as frequency and percentage. The chi-square test or Fisher's exact test was used to compare categorical variables among the three severity groups. A standard $t$-test or the Mann-Whitney $U$ test was used to compare continuous variables. All statistical analyses were performed using SPSS software. A two-sided $p$ value of $<0.05$ was considered statistically significant.
TABLE 1: Characteristics of the 250 patients with acute pancreatitis.

\begin{tabular}{|c|c|}
\hline Age in years & $58 \pm 17$ \\
\hline Male & $140(56)$ \\
\hline Comorbidities & $180(72)$ \\
\hline Hypertension & $117(47)$ \\
\hline Diabetes & $64(26)$ \\
\hline Dyslipidemia & $35(14)$ \\
\hline Malignancy & $29(12)$ \\
\hline Cardiovascular disease & $28(11)$ \\
\hline Chronic kidney disease & $16(6)$ \\
\hline Chronic obstructive pulmonary disease & $5(2)$ \\
\hline HIV/AIDS & $3(1)$ \\
\hline \multicolumn{2}{|l|}{ Department of admission } \\
\hline Medicine & $173(69)$ \\
\hline Surgery & $77(31)$ \\
\hline \multicolumn{2}{|l|}{ Signs and symptoms } \\
\hline Abdominal pain & $247(99)$ \\
\hline Back pain & $160(64)$ \\
\hline Altered consciousness & $2(1)$ \\
\hline Dyspnea & $1(0.4)$ \\
\hline Duration of symptoms in hours & $24(7-48)$ \\
\hline \multicolumn{2}{|l|}{ Serum pancreatic enzymes } \\
\hline Amylase $\geq 3$ times upper limit of normal & $229(95)$ \\
\hline Lipase $\geq 3$ times upper limit of normal & $212(95)$ \\
\hline Amylase and lipase $\geq 3$ times upper limits of normal & $196(91)$ \\
\hline Performance of CT & $62(25)$ \\
\hline Number of days after admission & $5(1-39)$ \\
\hline \multicolumn{2}{|l|}{ Etiology of AP } \\
\hline Gallstones & $113(45)$ \\
\hline Alcohol-related disease & $41(16)$ \\
\hline Post-ERCP & $35(14)$ \\
\hline Miscellaneous & $24(10)$ \\
\hline Idiopathic & $37(15)$ \\
\hline
\end{tabular}

Age and BMI are presented as mean \pm standard deviation; all other data are presented as $n$ (\%) or median (range). AP: acute pancreatitis; CT: computed tomography; ERCP: endoscopic retrograde cholangiopancreatography.

\section{Results}

3.1. Patients' Characteristics. The data of 272 consecutive patients with AP were reviewed. Twenty-two patients were excluded due to incomplete data. In total, 250 patients with AP met the eligibility criteria. One-hundred forty patients (56\%) were male, and 110 patients (44\%) were female. The mean age of the patients was $58 \pm 17$ years ( $56 \pm 16$ years in male and $62 \pm 19$ years in female), and $72 \%$ had various comorbidities (Table 1). Almost all patients (99\%) presented with abdominal pain, $64 \%$ had referred pain to the back, and $1 \%$ presented with altered consciousness. The median duration of symptoms was 24 hours before admission, of which was not different between male (median 24 hours, range 648 hours) and female (median 24 hours, range 8-48 hours). Both the serum amylase and lipase levels had a sensitivity of $95 \%$ for a diagnosis of AP, and the combination of both 
TABLE 2: Etiology of acute pancreatitis according to the gender.

\begin{tabular}{lccc}
\hline Etiology, $n(\%)$ & Male $(n=140)$ & Female $(n=110)$ & $p$ value \\
\hline Gallstone & $47(33.6)$ & $67(60.9)$ & $<0.001$ \\
Alcohol & $36(25.7)$ & $5(4.5)$ & $<0.001$ \\
Post-ERCP & $19(13.6)$ & $16(14.5)$ & 0.823 \\
Idiopathic & $23(16.4)$ & $14(12.7)$ & 0.413 \\
\hline
\end{tabular}

All data are presented as $n$ (\%). ERCP: endoscopic retrograde cholangiopancreatography.

enzymes was not superior to the use of either enzyme alone. One-fourth of the patients underwent $\mathrm{CT}$ at a median of 5 days after admission.

The etiologies of AP were gallstones (45\%), alcoholrelated disease (16\%), postendoscopic retrograde cholangiopancreatography (ERCP) (14\%), idiopathic (15\%), and miscellaneous (10\%). When comparing the etiology of AP in women to those in men, women had significantly more gallstone pancreatitis (60.9\% versus $33.6 \%)$, but less alcoholic pancreatitis (4.5\% versus $25.7 \%$ ) (Table 2 ).

3.2. Severity of AP. The severity of AP according to the 2012 revision of the Atlanta classification was mild, moderately severe, and severe in $72 \%, 16 \%$, and $12 \%$ of patients, respectively (Figure 1). Eighty-two percent of patients had no OF, $6 \%$ had transient OF, and $12 \%$ had persistent OF.

3.3. Accuracy of Early Severity Assessment Tools to Predict Severe AP. Using the 2012 revision of the Atlanta classification as the gold standard, the sensitivity, specificity, positive predictive value, and negative predictive value of the two most commonly used severity assessment tools [bedside index for severity in AP (BISAP) score of $\geq 3$ and the presence of systemic inflammatory response syndrome (SIRS) upon admission] are shown in Table 3. The Acute Physiology and Chronic Health Evaluation II (APACHE II) score was evaluated in very few patients; thus, this score was not analyzed.

3.4. Intravenous Fluid. The two essential treatments of severe $\mathrm{AP}$ that are able to reduce OF and/or mortality are early fluid resuscitation and enteral feeding [9]. The mean total intravenous fluid resuscitation volume during the first 24 hours in the studied patients was 2720,3150 , and $4660 \mathrm{~mL}$ in patients with mild, moderately severe, and severe AP, respectively. The type of intravenous fluid was normal saline solution in approximately $60 \%$ of patients and Ringer's solution in approximately $30 \%$ (Figure 2).

3.5. Enteral Nutrition. All patients with mild and moderately severe AP and $77 \%$ of those with severe AP received enteral nutrition. The median time until the start of enteral feeding was 16,24 , and 48 hours in patients with mild, moderately severe, and severe AP, respectively. Among patients with mild AP, $97 \%$ received enteral nutrition orally and 3\% required nasogastric feeding. Among patients with moderately severe $\mathrm{AP}, 80 \%$ received enteral nutrition orally, $18 \%$ received it via a nasogastric tube, and only $2 \%$ received it via a nasojejunal tube. In contrast, $52 \%$ of patients with

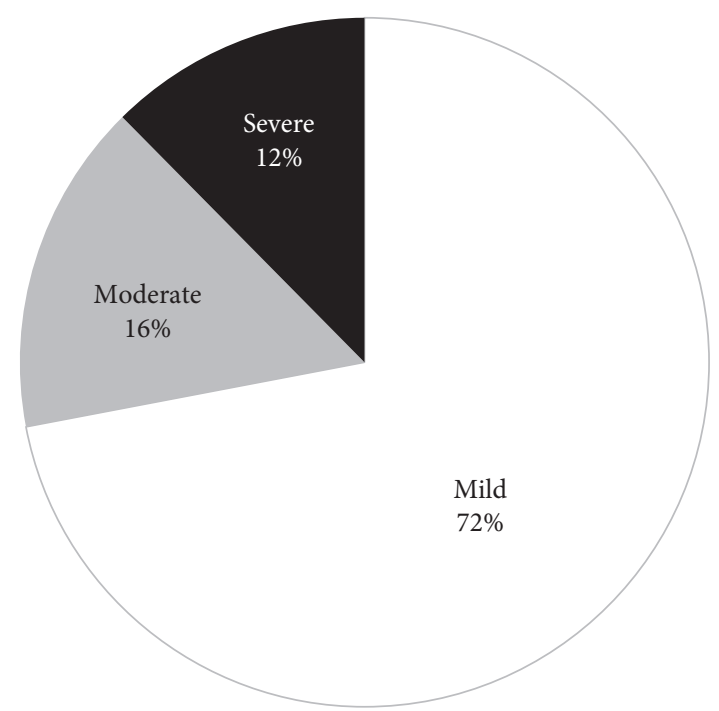

FIgURE 1: Severity of acute pancreatitis.

TABLE 3: Accuracy of the two most common methods to predict severe acute pancreatitis.

\begin{tabular}{lcccc}
\hline & $\begin{array}{c}\text { Sensitivity } \\
(\%)\end{array}$ & $\begin{array}{c}\text { Specificity } \\
(\%)\end{array}$ & $\begin{array}{c}\text { PPV } \\
(\%)\end{array}$ & $\begin{array}{c}\text { NPV } \\
(\%)\end{array}$ \\
\hline $\begin{array}{l}\text { SIRS upon } \\
\text { admission }\end{array}$ & 87 & 69 & 29 & 97 \\
BISAP score of $\geq 3$ & 61 & 90 & 46 & 94 \\
\hline
\end{tabular}

SIRS: systemic inflammatory response syndrome; BISAP: bedside index for severity in acute pancreatitis; PPV: positive predictive value; NPV: negative predictive value.

severe AP required nasogastric feeding, $23 \%$ could take it orally, and $3 \%$ required nasojejunal feeding (Figure 3 ).

3.6. Necrotizing Pancreatitis and Local Complications. NP occurred in 17 patients (7\%). Almost 70\% of cases of NP involved parenchymal necrosis with or without peripancreatic necrosis, while only $30 \%$ involved solely peripancreatic necrosis. Regarding the local complications, 27 patients (11\%) had acute peripancreatic fluid collections (APFC) and 13 patients (5\%) developed acute necrotic collections (ANC). Pancreatic pseudocysts were detected in 2 out of 4 patients with APFC, who had follow-up CT. Walled-off necrosis (WON) occurred in 9 out of 12 patients with necrotizing pancreatitis or ANC, who had follow-up CT (Table 4).

3.7. Pancreatic Infection. Overall, 5 out of 17 patients (29\%) with NP had infected necrosis. The diagnostic methods of infected necrosis were the presence of gas on CT $(66.6 \%)$ and Gram staining or culture of specimens obtained from fine-needle aspiration or percutaneous drainage (50.0\%). The median time until the diagnosis of pancreatic infection was 17 days (range, 7-25 days) after admission. All patients with pancreatic infections were treated by either percutaneous drainage (4 patients, $80 \%$ ) or open necrosectomy (1 patient, 20\%) (Table 5). 


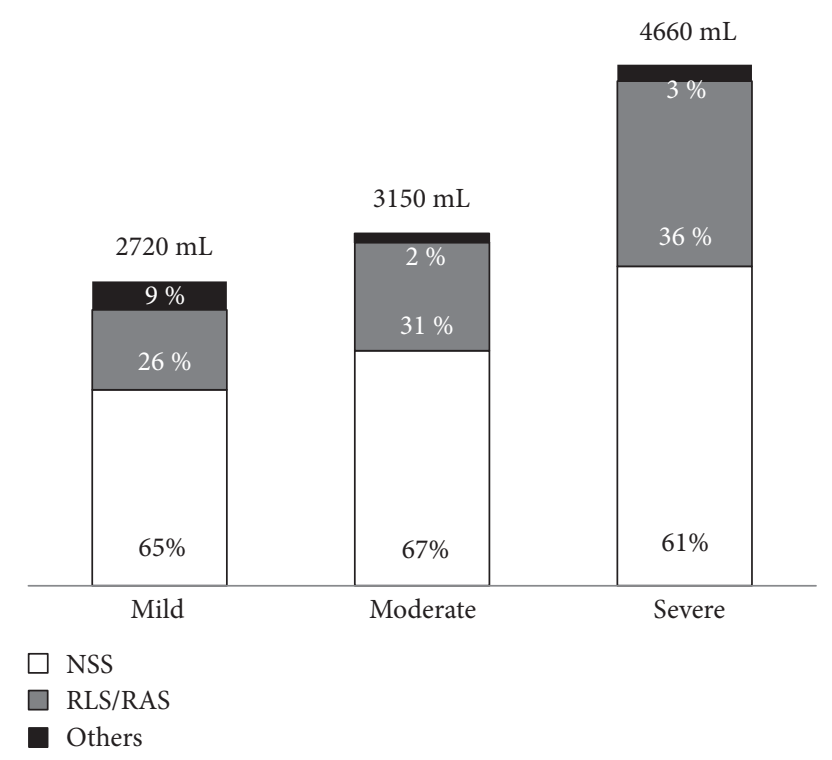

FIgURE 2: Types and amounts of intravenous fluid therapy during the first 24 hours. NSS, normal saline solution; RLS/RAS, Ringer's lactate solution/Ringer's acetate solution.

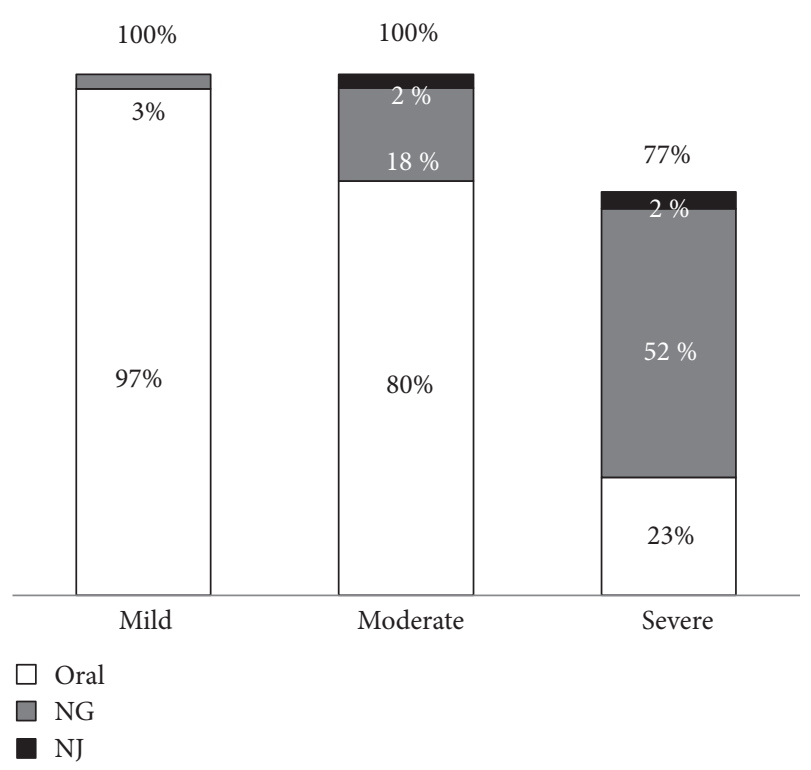

Figure 3: Routes of enteral nutrition. NG, nasogastric; NJ, nasojejunal.

3.8. Outcomes. The median length of hospital stay was 6,9 , and 13 days in the mild, moderately severe, and severe AP groups, respectively, with significant differences $(p<0.001)$. Surgery was performed in two patients with severe AP. One patient underwent pancreatic necrosectomy, and another underwent exploratory laparotomy due to a diagnosis of generalized peritonitis and was found to have AP. No surgery was performed in the mild and moderately severe groups. The AP-related mortality rates were $1 \%, 3 \%$, and $42 \%$ in the mild, moderately severe, and severe AP groups, respectively, with significant differences $(p<0.001)$ (Table 6). In
TABLE 4: Necrotizing pancreatitis and local complications of acute pancreatitis.

\begin{tabular}{lc}
\hline Necrotizing pancreatitis & \\
All & $17(7)$ \\
Parenchymal necrosis & $3(1)$ \\
Peripancreatic necrosis & $5(2)$ \\
Both & $9(4)$ \\
\hline Local complications & \\
Acute peripancreatic fluid collection & $27(11)$ \\
Acute necrotic collection & $13(5)$ \\
Pancreatic pseudocysts & $2(1)$ \\
Walled-off necrosis & $9(4)$ \\
\hline
\end{tabular}

Data are presented as $n(\%)$.

TABle 5: Pancreatic infection status of the 17 patients with necrotizing pancreatitis and their treatments.

\begin{tabular}{lc}
\hline Necrotizing pancreatitis & \\
\hline Sterile & $12(71)$ \\
Infected & $5(29)$ \\
$\quad$ Percutaneous drainage & 4 \\
Surgery & 1 \\
\hline
\end{tabular}

Data are presented as $n$ (\%).

the comparison of 13 patients with severe AP who died and 18 patients with severe AP who survived, there was no difference regarding the patients' age, gender, etiology of AP, timing of admission, the ICU care, type of intravenous fluid resuscitation, presence of local complications, and surgery (data not shown). The median ICU stays were 7 days and 8 days. The two deaths in the mild AP group had nonpancreatic causes (acute cholangitis with septicemia and upper gastrointestinal bleeding, resp.).

\section{Discussion}

The 2012 revision of the Atlanta classification is a recently proposed gold standard for the classification of AP [4]. The present study is among one of the few that have used the revised Atlanta classification to evaluate the severity, treatments, and outcomes of AP [10]. The results of this study are of importance and represent a milestone in the development of a guideline for AP in Thai patients. Our results highlight some important points regarding AP in Thai patients.

The age and sex of the patients with AP in this study were very similar to those of patients in recent studies from the West [11] and Japan [12]; however, the mean age was slightly higher than that in a study from China $[13,14]$. The typical clinical presentation was abdominal pain in almost all patients. Referred pain to the back was present in twothirds of the patients of the present study and in close to one-half of patients in another study [15].

The etiology of AP in this study was comparable with that in other studies from Asia [12,13], with the leading causes of AP being gallstones and alcohol-related disease. However, gallstones were more prevalent in female, while alcohol was 
TABle 6: Treatment outcomes.

\begin{tabular}{|c|c|c|c|c|}
\hline & Mild $(n=179)$ & Moderately severe $(n=40)$ & Severe $(n=31)$ & $p$ \\
\hline Length of stay in days & $6(1-29)$ & $9(3-147)$ & $13(1-110)$ & $<0.001$ \\
\hline Surgery & $0(0)$ & $0(0)$ & $2(100)$ & 0.015 \\
\hline Mortality & $2(1)$ & $1(3)$ & $13(42)$ & $<0.001$ \\
\hline
\end{tabular}

Data are presented as median (range) or $n$ (\%).

so in male, of which were well known. The incidence of gallstone pancreatitis was not different from that in our previous study in 2007 [7]; however, the incidence of alcohol-related AP was significantly lower with a paradoxical increase in post-ERCP pancreatitis, which was the fourth most common etiology in the present study. This can be explained by the increasing numbers of ERCP procedures performed during the last decade in our institution, which is a tertiary hospital. Idiopathic AP was the third most common etiology in the present study; it fell within the acceptable range $(\leq 20 \%)$ suggested by most guidelines [9, 16-19].

The present study demonstrated that the severity of AP in our institution according to the 2012 revision of the Atlanta classification was mild, moderately severe, and severe in $72 \%, 16 \%$, and $12 \%$ of patients, respectively. These data are consistent with recent data from Western studies, which reported mild AP in $70 \%$ to $85 \%$ of patients, moderately severe AP in $10 \%$ to $15 \%$ of patients, and severe AP in $5 \%$ to $15 \%$ of patients $[20,21]$. Very few data are available from Asia because most studies were published before the endorsement of the 2012 revision of the Atlanta classification [12]. However, a recent study from China [22] that utilized the 2012 revision of the Atlanta classification reported mild, moderate, and severe AP in $30 \%, 53 \%$, and $17 \%$ of patients, respectively. The major difference between that study and ours occurred among the patients with moderately severe AP; the incidence of both OF and local complications differed considerably between these two studies. The reason for the very high prevalence of moderately severe AP in this Chinese study is unclear, but we feel that it is too high when compared with those in the other studies from Western countries $[20,21]$. It is possible that the Chinese study did not exclude transferred patients, and therefore, the rates of moderate and severe AP were higher. Our study had a strength that we excluded transferred patients and, therefore, would represent the more accurate picture of the severity of AP. Another possible reason for the lower prevalence of moderately severe AP in our study might be the lack of CT examination in all patients. Our CT examinations were mainly performed in patients suspected to have severe AP, as reflected by the $25 \%$ frequency of CT in our study. Some patients with mild AP might have actually had local complications and would have been classified as having moderately severe AP if CT had been performed. However, our practice of performing CT complied well with all standard guidelines of AP $[9,19,23]$, which recommend CT mainly for patients with clinically severe AP.

The use of early severity assessment tools for AP is another interesting topic. A recent meta-analysis [24] and a comprehensive review by the present authors [25] concluded that the four best tools with which to predict the severity of
AP within the first 24 hours were the presence of SIRS and the APACHE II, BISAP, and Japanese severity scale scores upon admission. All of these parameters have an advantage of a very high negative predictive value with which to rule out severe AP $[24,25]$. In our institute, the presence of SIRS and the BISAP score are frequently used, whereas the APACHE II score and the Japanese severity scale score are rarely used. The present study confirmed that the presence of SIRS and the BISAP score on admission did very well in ruling out severe AP due to their high NPV (94\%-97\%). The presence of SIRS on admission was slightly more effective than the BISAP score in excluding severe AP.

Fluid resuscitation during the first 24 hours and enteral nutrition are currently the two most critical and essential treatments of AP because they can reduce both OF and mortality [26]. In the present study, the intravenous fluid volume administered to patients with severe AP complied well with the guidelines, which recommend administration of 3 to $5 \mathrm{~L}$ of fluid during the first 24 hours $[9,19,23]$. However, we found that instead of using Ringer's solution, which is preferred by most guidelines $[9,19,23]$, most of our patients received normal saline solution. The reason for this difference is unknown, but we hypothesized that it might be related to a lack of knowledge among physicians or a more familiarity with normal saline solution than Ringer's solution. This problem is critical and must be urgently solved systematically by providing education to the physicians or making a clinical institute policy. Conversely, enteral nutrition, which is the most effective treatment for AP and is endorsed by all guidelines $[9,19,23]$, was prescribed to almost all patients in the present study.

Regarding necrotizing pancreatitis and local complications of AP, an interesting finding was that NP was not prevalent. The $7 \%$ frequency of NP in the present study was much lower than the $10 \%$ to $20 \%$ frequency in most studies. The exact reason for this difference is unknown. However, we hypothesize that it might be due to the undiagnosed pancreatic necrosis, particularly in patients with clinically mild AP, because CT usually was not done in such patients. Further study to determine the presence of moderately severe AP (from local complications) in patients with clinically mild $\mathrm{AP}$ in our country is required. Another possible reason could be the misclassification of some ANC as APFC, particularly during the first week of the disease because majority of the CT in the present study were performed within the first week. It is also the possible reason for the very low incidence of late local complications of AP, pseudocyst, and WON in the present study because we did not have a systematic follow-up CT for patients who had APFC and ANC. Therefore, we might underdiagnose many pseudocyst and WON. 
The major outcomes of AP in the present study were similar to those in most guidelines [2]. The median hospital stay in our study was 6 days $(6,9$, and 13 days for mild, moderate, and severe AP, respectively), which is similar to that reported in other studies [22]. Surgery was rare and was only performed in 2 of 250 patients. The mortality of AP in the present study was within the range reported in standard guidelines [2] and another recent study [22]. However, the $42 \%$ mortality rate of severe AP in this study was slightly higher than it should be (20-30\%) [2]. We could not find any reasonable explanation for this high mortality, including the timing of admission, patients' age, ICU admission, type of intravenous fluid, enteral feeding, presence of local complications, or surgery. Finally, we could not deny that it might be due to the quality of our intensive care. Thus, this is another opportunity for improvement. Normally, mild AP should have a mortality rate near $0 \%$. In the present study, however, two patients (1\%) with mild AP died. The causes of death were cholangitis with severe Pseudomonas aeruginosa septicemia and upper gastrointestinal bleeding, respectively, neither of which was directly caused by AP.

The strength of the present study is that it is the first comprehensive study on AP in Thai patients. The "gold standard" 2012 revision of the Atlanta classification was used. All CT scans were reviewed by experts in pancreatic disease according to the 2012 revision of the Atlanta classification, and all treatments aligned with all recent guidelines with no limitations of the facilities. However, the weaknesses of this study are the retrospective and observational nature of the study and the study was conducted in a university hospital setting; therefore, the results might not accurately represent the clinical picture of AP in Thailand. Additionally, the etiology of AP was post-ERCP in $14 \%$ of the patients in this study, which would not be found in the community setting. Nevertheless, when we calculated the severity of AP after excluding the 35 patients with post-ERCP pancreatitis, the results were unchanged (data not shown).

\section{Conclusions}

The severity of AP in our study was mild, moderately severe, and severe in $72 \%, 16 \%$, and $12 \%$ of patients, respectively. NP was not prevalent, and infected necrosis occurred in one-third of NP. Mortality was high in severe AP. Most treatments of AP, particularly the amount of intravenous fluid administration and enteral feeding, complied well with the standard guidelines except the use of normal saline solution rather than Ringer's solution. Further strategies to improve the intensive care quality of severe $\mathrm{AP}$ and to endorse the use of Ringer's solution are urgently needed. Prospective multicenter data collection is also advocated.

\section{Conflicts of Interest}

The authors declare that there is no conflict of interests regarding the publication of this paper.

\section{References}

[1] E. L. Bradley 3rd, "A clinically based classification system for acute pancreatitis. Summary of the International Symposium on Acute Pancreatitis, Atlanta, Ga, September 11 through 13, 1992," Archives of Surgery, vol. 128, no. 5, pp. 586-590, 1993.

[2] P. A. Banks, M. L. Freeman, and G. Practice Parameters Committee of the American College of, "Practice guidelines in acute pancreatitis," The American Journal of Gastroenterology, vol. 101, no. 10, pp. 2379-2400, 2006.

[3] S. Pongprasobchai, "Acute pancreatitis: evidence-based management," Thai Journal of Gastroenterology, vol. 5, no. 2, pp. 111-122, 2004.

[4] P. A. Banks, T. L. Bollen, C. Dervenis et al., "Classification of acute pancreatitis-2012: revision of the Atlanta classification and definitions by international consensus," Gut, vol. 62, no. 1, pp. 102-111, 2013.

[5] C. Pramoolsinsap and S. Kurathong, "Pancreatitis: an analysis of 106 patients admitted to Ramathibodi Hospital during 1969-1984," Journal of the Medical Association of Thailand, vol. 72, no. 2, pp. 74-81, 1989.

[6] P. Navicharern, W. Wesarachawit, S. Sriussadaporn et al., "Management and outcome of severe acute pancreatitis," Journal of the Medical Association of Thailand, vol. 89, Supplement 3, pp. S25-S32, 2006.

[7] S. Pongprasobchai, R. Thamcharoen, and S. Manatsathit, "Changing of the etiology of acute pancreatitis after using a systematic search," Journal of the Medical Association of Thailand, vol. 92, Supplement 2, pp. S38-S42, 2009.

[8] S. Pongprasobchai, V. Jianjaroonwong, P. Charatcharoenwitthaya et al., "Erythrocyte sedimentation rate and Creactive protein for the prediction of severity of acute pancreatitis," Pancreas, vol. 39, no. 8, pp. 1226-1230, 2010.

[9] IAP/APA Acute Pancreatitis Guidelines Working Group, "IAP/APA evidence-based guidelines for the management of acute pancreatitis," Pancreatology, vol. 13, no. 4, Supplement 2, pp. e1-15, 2013.

[10] N. G. Acevedo-Piedra, N. Moya-Hoyo, M. Rey-Riveiro et al., "Validation of the determinant-based classification and revision of the Atlanta classification systems for acute pancreatitis," Clinical Gastroenterology and Hepatology, vol. 12, no. 2, pp. 311-316, 2014.

[11] H. Birgisson, P. H. Moller, S. Birgisson et al., "Acute pancreatitis: a prospective study of its incidence, aetiology, severity, and mortality in Iceland," The European Journal of Surgery, vol. 168, no. 5, pp. 278-282, 2002.

[12] S. Hamada, A. Masamune, K. Kikuta et al., "Nationwide epidemiological survey of acute pancreatitis in Japan," Pancreas, vol. 43, no. 8, pp. 1244-1248, 2014.

[13] Y. Bai, L. Jia, B. Wang et al., "Acute pancreatitis in the Guangdong Province, China," Digestion, vol. 75, no. 2-3, pp. 74-79, 2007.

[14] Y. J. Gao, Y. Q. Li, Q. Wang et al., "Analysis of clinical features of acute pancreatitis in Shandong Province, China," Journal of Gastroenterology and Hepatology, vol. 22, no. 3, pp. 340-344, 2007.

[15] P. A. Banks, "Acute pancreatitis: diagnosis," in Pancreatitis, P. G. Lankisch and P. A. Banks, Eds., pp. 75-116, SpringerVerlag, Berlin, 1998. 
[16] Working Party of the British Society of Gastroenterology, "UK guidelines for the management of acute pancreatitis," Gut, vol. 54, Supplement 3, pp. iiil-iii9, 2005.

[17] C. E. Forsmark and J. Baillie, "AGA Institute technical review on acute pancreatitis," Gastroenterology, vol. 132, no. 5, pp. 2022-2044, 2007.

[18] R. Pezzilli, G. Uomo, A. Zerbi et al., "Diagnosis and treatment of acute pancreatitis: the position statement of the Italian Association for the study of the pancreas," Digestive and Liver Disease, vol. 40, no. 10, pp. 803-808, 2008.

[19] S. Tenner, J. Baillie, J. DeWitt, S. S. Vege, and G. American College of, "American College of Gastroenterology guideline: management of acute pancreatitis," The American Journal of Gastroenterology, vol. 108, no. 9, pp. 1400-1415, 2013.

[20] T. L. Bollen, V. K. Singh, R. Maurer et al., "A comparative evaluation of radiologic and clinical scoring systems in the early prediction of severity in acute pancreatitis," The American Journal of Gastroenterology, vol. 107, no. 4, pp. 612-619, 2012.

[21] R. Mounzer, C. J. Langmead, B. U. Wu et al., "Comparison of existing clinical scoring systems to predict persistent organ failure in patients with acute pancreatitis," Gastroenterology, vol. 142, no. 7, pp. 1476-1482, 2012.

[22] Y. Chen, L. Ke, Z. Tong, W. Li, and J. Li, “Association between severity and the determinant-based classification, Atlanta 2012 and Atlanta 1992, in acute pancreatitis: a clinical retrospective study," Medicine (Baltimore), vol. 94, no. 13, article e638, 2015.

[23] M. Yokoe, T. Takada, T. Mayumi et al., "Japanese guidelines for the management of acute pancreatitis: Japanese guidelines 2015," Journal of Hepato-Biliary-Pancreatic Sciences, vol. 22, no. 6, pp. 405-432, 2015.

[24] C. J. Yang, J. Chen, A. R. Phillips, J. A. Windsor, and M. S. Petrov, "Predictors of severe and critical acute pancreatitis: a systematic review," Digestive and Liver Disease, vol. 46, no. 5 , pp. $446-451,2014$.

[25] S. Pongprasobchai, "Use of prognostic markers in the management of acute pancreatitis," Thai Journal of Gastroenterology, vol. 15, no. 2, pp. 62-68, 2014.

[26] M. Al-Omran, Z. H. Albalawi, M. F. Tashkandi, and L. A. Al-Ansary, "Enteral versus parenteral nutrition for acute pancreatitis," Cochrane Database of Systematic Reviews, no. 1, article CD002837, 2010. 


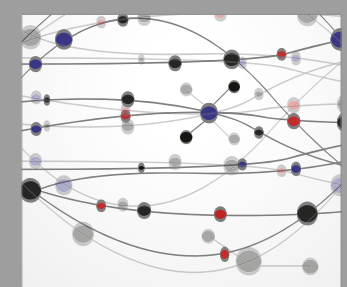

The Scientific World Journal
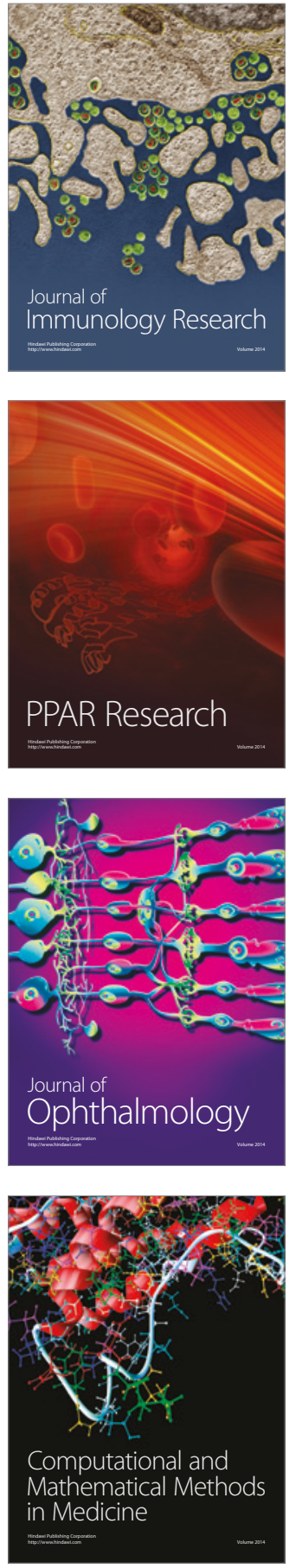

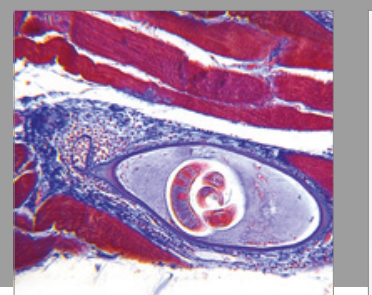

Gastroenterology Research and Practice
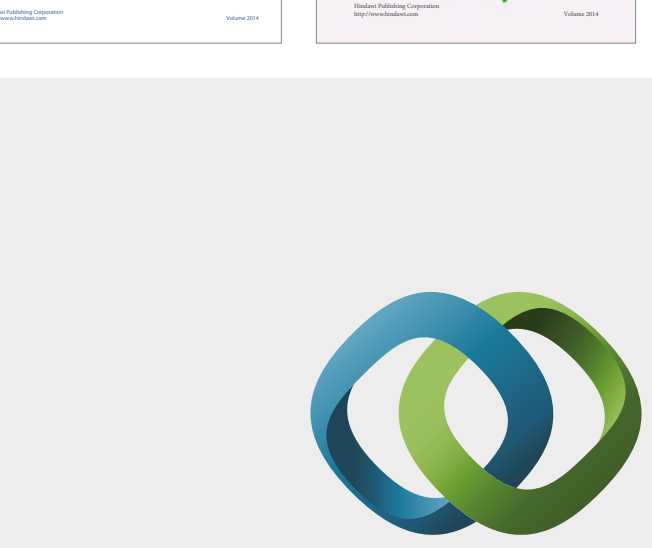

\section{Hindawi}

Submit your manuscripts at

https://www.hindawi.com
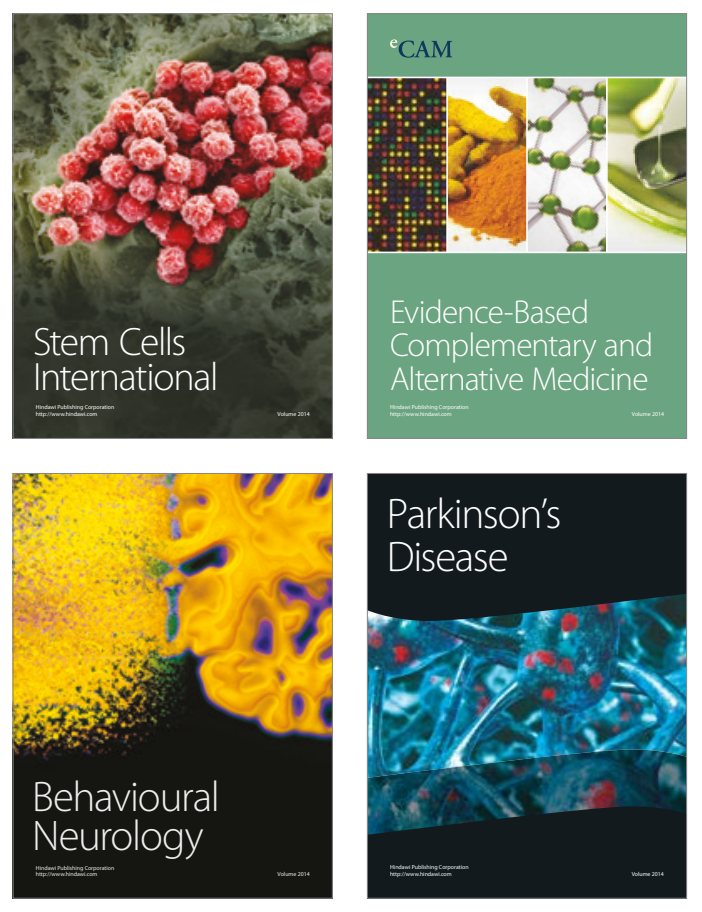
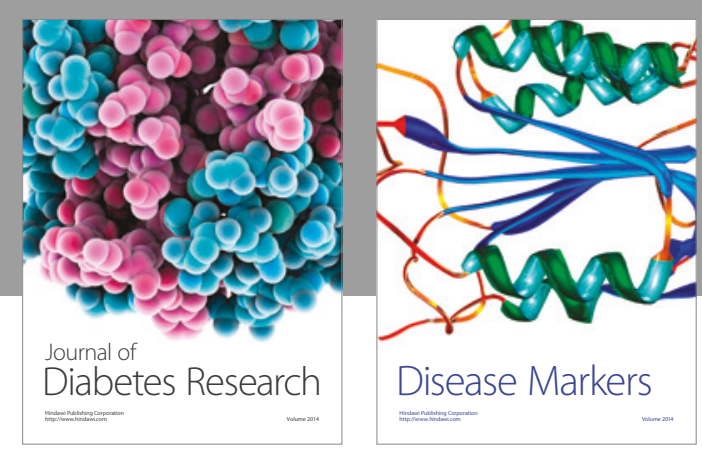

Disease Markers
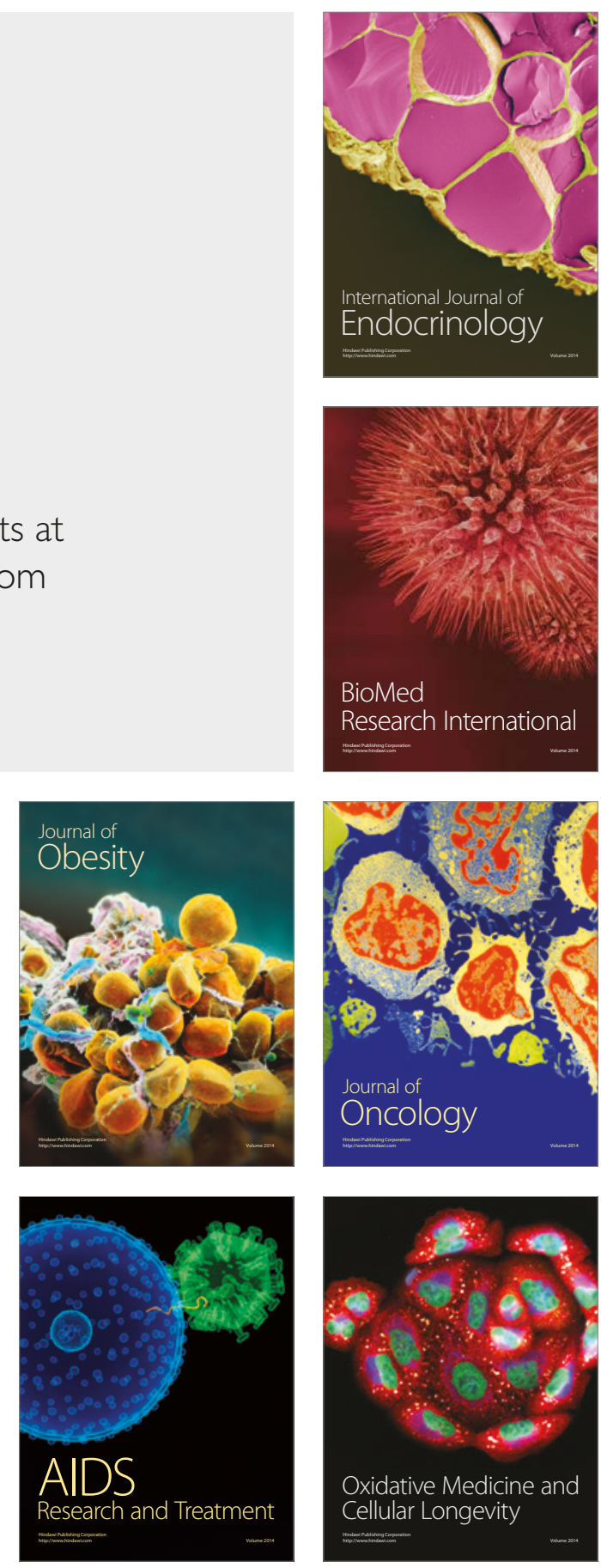